\title{
Prevalence of Rotavirus in Under 5 Year Old Children Hospitalized for Acute Gastroenteritis, Swaziland, 2013 - 2014
}

\author{
Gugu Maphalala ${ }^{1}$, Goitom Weldegebriel ${ }^{2,}$, Nomcebo Phungwayo $^{1}$, Eunice Ruhinda ${ }^{3}$, \\ Njabulo Lukhele ${ }^{3}$, Getahun Tsegaye ${ }^{4}$, Gilbert Masona ${ }^{4}$, Nomsa Nomcebo Dube ${ }^{5}$, \\ Nonhlanhla Dlamini $^{5}$, Angel Dlamini ${ }^{5}$, Philile Shabangu ${ }^{6}$, Lonkululeko Khumalo ${ }^{6}$ \\ ${ }^{1}$ National Reference Laboratory, Swaziland Health Laboratory Services, Mbabane, Swaziland \\ ${ }^{2}$ World Health Organization, Inter country support team for East and Southern Africa, Zimbabwe, Harare \\ ${ }^{3}$ Mbabane Government Hospital, Mbabane, Swaziland \\ ${ }^{4}$ Raleigh Fitkin Memorial Hospital, Manzini, Swaziland \\ ${ }^{5}$ Ministry of Health, EPI, Mbabane, Swaziland \\ ${ }^{6}$ World Health Organization, Mbabane, Swaziland
}

\section{Email address:}

gpmaph@gmail.com (G. Maphalala), weldegebrielg@who.int (G. Weldegebriel), cebophung@gmail.com (N. Phungwayo), enyesire@yahoo.com (E. Ruhinda), lomav@yahoo.com (N. Lukhele), getahun@realnet.co.sz (G. Tsegaye), gmasona@gmail.com (G. Masona), nomsamq@gmail.com (N. N. Dube), Dlamininhlanhla839@yahoo.com (N. Dlamini), dlaminiangel@yahoo.com (A. Dlamini), shabangup@who.int (P. Shabangu),khumalol@who.int (L. Khumalo)

${ }^{*}$ Corresponding author

\section{To cite this article:}

Gugu Maphalala, Goitom Weldegebriel, Nomcebo Phungwayo, Eunice Ruhinda, Njabulo Lukhele, Getahun Tsegaye, Gilbert Masona, Nomsa Nomcebo Dube, Nonhlanhla Dlamini, Angel Dlamini, Philile Shabangu, Lonkululeko Khumalo. Prevalence of Rotavirus in Under 5 Year Old Children Hospitalized for Acute Gastroenteritis, Swaziland, 2013 - 2014. Science Journal of Public Health. Vol. 5, No. 4, 2017, pp. 353-358. doi: 10.11648/j.sjph.20170504.22

Received: May 4, 2017; Accepted: May 16, 2017; Published: July 26, 2017

\begin{abstract}
Background: Rotavirus is the most common cause of severe diarrhoea among children $<5$ years of age worldwide. To determine the prevalence of severe rotavirus infection in children admitted with acute gastroenteritis attending Mbabane Government Referral Hospital in the Hhohho region and Raleigh Fitkin Memorial (RFM) Hospital in the Manzini Region of Swaziland, sentinel surveillance was conducted in January 2013 - December 2014. Methods: This is hospital based sentinel surveillance study. A total of 331 children under the age of five years with acute diarrhoea that were admitted to the hospitals were enrolled into the sentinel surveillance and 302 cases had stool samples tested for rotavirus antigens by enzyme immunoassay Pro Spec T Rotavirus kit. The G and P genotypes were established by multiplex nested reverse transcription polymerase chain reaction. Results: Rotavirus was detected in $159(52.6 \%)$ of the 302 children with acute diarrhoea that had stool specimens collected and $108(68 \%)$ of positive cases of rotavirus gastroenteritis were between 6-11 months of age and 91\% were below the age of two years. Rotavirus infection occurred with peaks between June - August. During the $2013-2014$ period, 146 samples were subjected for reverse transcription polymerase chain reaction and genotyping assays. The most common genotypes detected in Swaziland were 82\% G2P [4] in 2013 and 93\% G1P [8] in 2014. Conclusion: Swaziland would benefit by introducing rotavirus vaccine and hence reduce the hospitalization burden of managing acute diarrhoea cases attributed to Rotavirus. The data from the sentinel hospitals can also be used to monitor the effectiveness of the vaccine once introduced.
\end{abstract}

Keywords: Diarrhoea, Rotavirus, Prevalence, Genotypes, Swaziland 


\section{Introduction}

Worldwide, $10 \%$ of under 5 years old mortality is caused by diarrhoeal diseases [1]. Rotavirus is one of the four common causes of moderate to severe diarrhoea among children under 5 years of age in Sub Saharan Africa and Asia [2]. Among other etiological agents, group A Rotavirus is the main cause of severe gastroenteritis in children less than 5 years of age worldwide and are associated with an estimate of 453,000 deaths globally [3]. In 2008, of the estimated 453,000 global deaths from rotavirus diarrhoea in children $<5$ years of age, more than half $(\mathrm{N}=230,000)$ occurred in African children [4].

Sentinel hospital based surveillance in many countries showed that rotavirus accounted for $21 \%-56 \%$ of diarrhoeal hospitalizations among children $<5$ years of age in Africa during the pre-vaccine era [5 - 14].

There is no Rotavirus data from a systematic study published from Swaziland that showed the prevalence of Rotavirus diarrhoea. The World Health Organization Regional office for Africa in collaboration with the Ministry of Health of Swaziland undertook the current hospital based sentinel surveillance since 2013 to assess the magnitude of Rotavirus infections among hospitalized children $<5$ years of age with acute watery diarrhoea in two hospitals and to generate data that can guide decisions on introduction of Rotavirus vaccine.

Rotavirus vaccines represent an important preventive approach to reducing rotavirus infection and offer the best hope for control of these ubiquitous infections [16]. In 2009, WHO recommended the use of rotavirus vaccine in all countries globally and particularly those countries with high mortality due to diarrhoea [17].

\section{Materials and Methods}

Active hospital based rotavirus surveillance for diarrhoea was conducted in 2 sentinel hospital sites (Mbabane Government Referral Hospital and Raleigh Fitkin Memorial (RFM) Hospital). Both are located in Urban areas and had a bed capacity of 575 and 350 respectively. Mbabane Government referral hospital serves 86, 647populations and RFM serves 141,244 people; these health facilities also serves as referral for other hospitals in the country thus increasing the catchment areas.

Children under the age of five years who presented with acute gastroenteritis (AGE), the occurrence of $>3$ episodes of diarrhoea (stools of less formed character than usual) within 24 hour period of $<7$ days duration as a primary illness and were admitted to a hospital ward or treated at the emergency unit at 1 of the 2 sentinel hospitals during the period Jan 2013 - December 2014, were enrolled in the surveillance program. Stool specimens were collected as in the standard guidelines [15]. According to the WHO AFRO guideline, children $>5$ years of age, with bloody diarrhoea, with symptoms $>7$ days or who acquired AGE during hospitalization for treatment of other illness were excluded. All information regarding inclusion criteria and basic demographic and clinical information for each case was captured on the standard rotavirus case investigation form.

\subsection{Laboratory Methods}

\subsubsection{Enzyme Immunoassay (EIA)}

A total of 302 stool specimens were screened for the presence of group a rotavirus antigen using the commercially available Pro Spec TTM Rotavirus enzyme immunoassay kit (Oxoid Ltd, UK) according to the manufacturer's instructions. Samples were prepared following manufacturer's protocol.

\subsubsection{Nucleic Acid Extraction}

Viral RNA from all EIA positive specimens was extracted from $140 \mu \mathrm{l}$ of $10 \%$ stool suspensions using the QIAamp viral RNA Mini kit (Qiagen, Inc., Valencia, CA USA) according to manufacturer's instructions and stored at $-80^{\circ} \mathrm{C}$.

\subsection{Genotyping by Multiplex RT-PCR This Is Correct}

The RNA extracts were subjected to multiplexed seminested reverse transcription-polymerase chain reaction (RTPCR). Two genes, VP7 (896 or 1061 bp) and VP4 (876 bp) were reverse-transcribed and amplified with primers SBeg9/End9 (Gouvea et al., 1990) and Con3/Con2 (Gentsch et al., 1992). Reverse transcription of doublestranded RNA (dsRNA) was carried out with the One Step RT-PCR Kit (Qiagen, Inc., Valencia, CA USA). After 5 min denaturation at $97^{\circ} \mathrm{C}$, the RNA was mixed with kit reagents and incubated at $42^{\circ} \mathrm{C}$ for 30 min to obtain complementary DNA (cDNA), immediately followed by the PCR reaction. These first round RT-PCR products were then used in a semi-nested PCR to identify G and P types (Gentsch et al., 1992). All PCR products were identified by electrophoresis in $2 \%$ agarose gels containing ethidium bromide and visualized under UV illumination. Genotype specific primers were used to confirm mixed infection and each amplicon generated was sequenced.

\section{Results}

Between January 2013 - December 2014, a total of 331 children (50.5\% males and $49.5 \%$ females) below the age of five years were hospitalized for management of acute gastroenteritis (AGE) in the two sentinel site hospitals. All cases had stool samples collected, of which $302(91 \%)$ were tested for Rotavirus. Of the total samples tested, $159(52.6 \%)$ were positive for Rotavirus; in 2013, rotavirus positives were $41.5 \%$ and rotavirus positivity was higher $(59.8 \%)$ in 2014 (Table 1). There were more females $(52.8 \%)$ that were positive for Rotavirus than males (47.2\%). 
Table 1. Under 5 years acute gastroenteritis (AGE) hospitalizations and rotavirus positives by year, by sentinel hospital site, Swaziland, 2013 - 2014.

\begin{tabular}{|c|c|c|c|c|c|c|c|c|c|}
\hline & Year 2 & & & Year 2 & & & & & \\
\hline $\begin{array}{l}\text { Hospital Site } \\
\text { Name }\end{array}$ & $\begin{array}{l}\text { AGE } \\
\text { cases }\end{array}$ & $\begin{array}{l}\text { Samples } \\
\text { tested }\end{array}$ & $\begin{array}{l}\text { Rotavirus } \\
\text { positives (\%) }\end{array}$ & $\begin{array}{l}\text { AGE } \\
\text { cases }\end{array}$ & $\begin{array}{l}\text { Samples } \\
\text { tested }\end{array}$ & $\begin{array}{l}\text { Rotavirus } \\
\text { positives (\%) }\end{array}$ & $\begin{array}{l}\text { Total AGE } \\
\text { cases }\end{array}$ & $\begin{array}{l}\text { Total samples } \\
\text { tested }\end{array}$ & Positives (\%) \\
\hline $\begin{array}{l}\text { MBABANE } \\
\text { HOSPITAL }\end{array}$ & 82 & 79 & $36(45.6 \%)$ & 98 & 93 & $62(66.7 \%)$ & 180 & 172 & $98(57 \%)$ \\
\hline RFM & 60 & 39 & $13(33.3 \%)$ & 91 & 91 & 48 (52.7\%) & 151 & 130 & $61(47 \%)$ \\
\hline Total & 142 & 118 & $49(41.5 \%)$ & 189 & 184 & $11059.8 \%)$ & 331 & 302 & $159(52.6 \%)$ \\
\hline
\end{tabular}

Case fatality among children admitted for acute gastroenteritis (AGE) was 9/231 (2.7\%). For rotavirus positives, case fatality was 2/159 (1.3\%) and the two rotavirus positive deaths occurred in 2014.

Acute gastroenteritis (AGE) hospitalizations were common among under five children and mainly affected children by AGE $(94.4 \%)$ were children below the age of 2 years (Figure 1$)$.

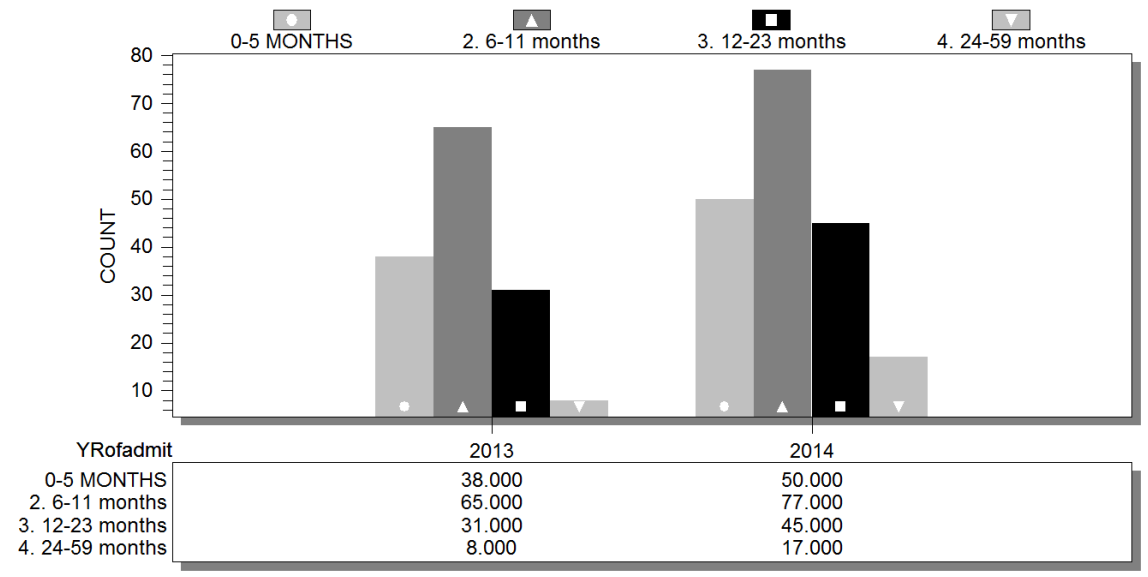

Figure 1. Diarrhoea hospitalizations Under 5 years by age group, Swaziland, 2013-2014.

Similarly, Rotavirus infection was common among $0-11$ months with $108(68 \%)$ positive and $145(91 \%)$ of the Rotavirus positives were below the age of 2 years (Figure 2). There was no statistically significant difference on rotavirus positivity by gender $(\mathrm{P}=.63,95 \%$ Confidence Interval 0.53 - 1.3). Among the Rotavirus positives, 53.5\% had severe dehydration, $34 \%$ had some dehydration and $0.6 \%$ was in shock. Treatments given for rehydration were mostly Intravenous fluid (IVF) $(84.3 \%)$, oral rehydration salt (ORS) $6.9 \%$ and both ORS and IVF in $7.5 \%$ of the cases.

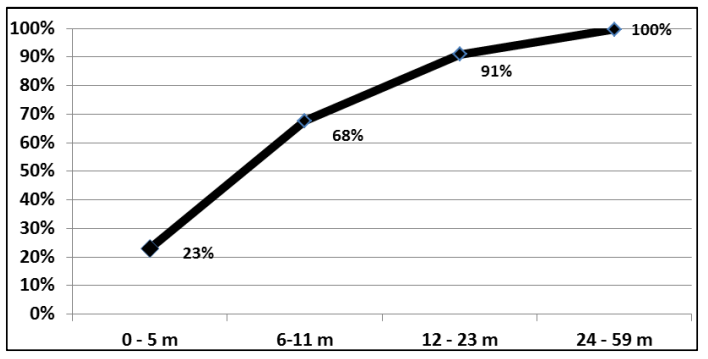

Figure 2. Cumulative age distribution of Rotavirus diarrhoea hospitalizations, 2013 - 2014, Swaziland.

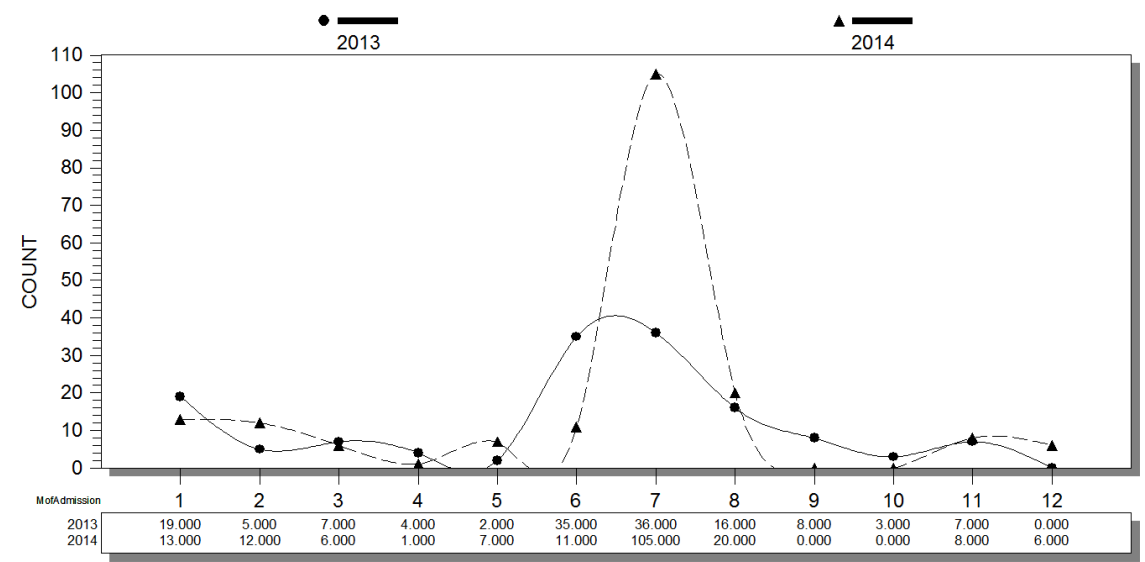

Figure 3. Seasonality of AGE hospitalizations in under 5 yrs olds, Swaziland, 2013-2014. 
Diarrhoea hospitalizations among under five year olds occurred all year round with the highest numbers seen between June - August (Figure 3).

The peak rotavirus season coincided with the peak diarrhoeal season during the cool dry season period where more than $90 \%$ of the cases occurred (Figure 4 ).

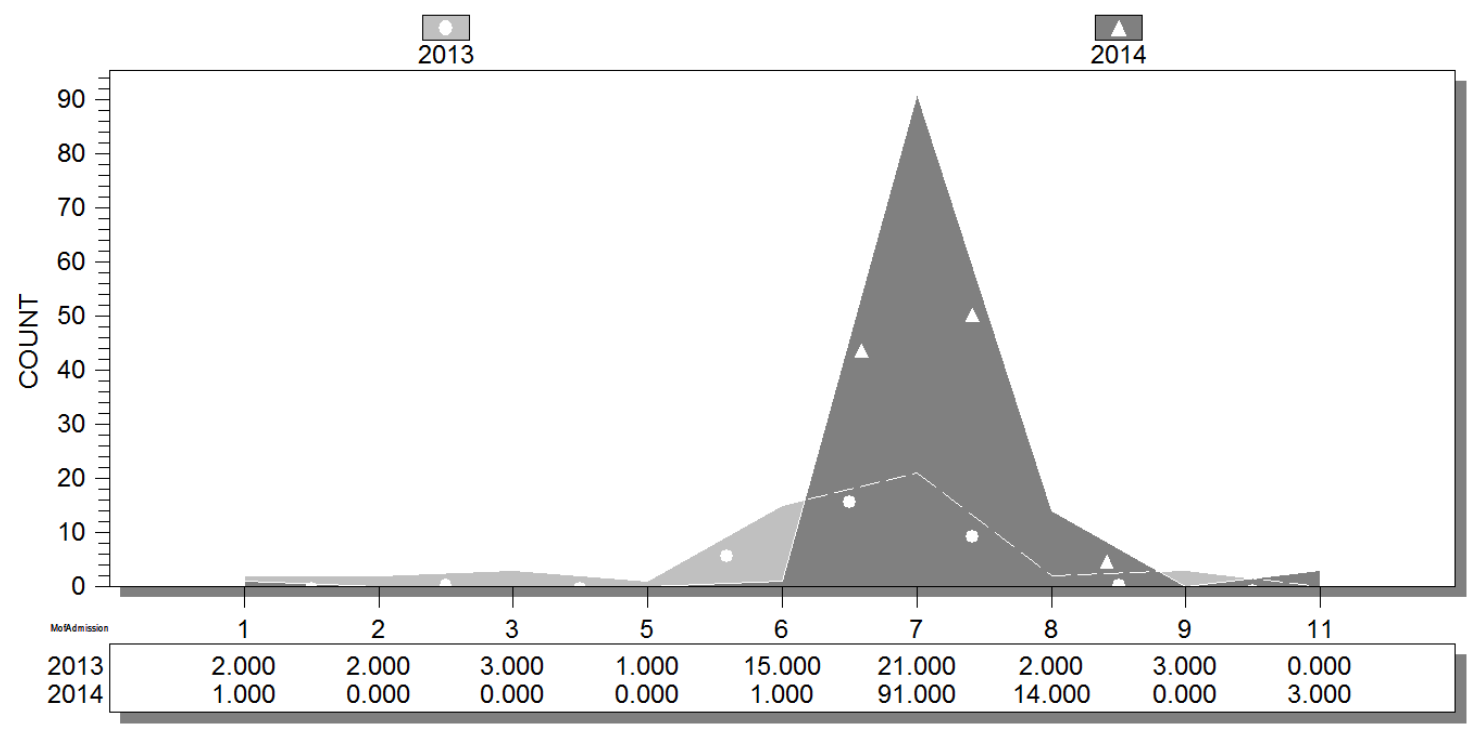

Figure 4. Seasonality of Rotavirus diarrhoea hospitalizations, 2013 - 2014, Swaziland.

Genotyping analyses were carried out to determine the $G$ (VP7) and P (VP4) rotavirus strains which were circulation in the 2 sentinel sites. All 166 EIA positive were subjected to both VP4 and VP7 gene genotyping in order to determine the $P$ and $G$ types of all specimens. A total of 148 (89\%) specimens were successfully genotyped; among these 52 specimens were genotyped for year 2013 and 96 for year 2014 as indicated on figure 5 below. In 2013, G2P [4] (82\%) were the most predominant genotypes followed by the, G4P [14] (4\%) and then GIP [8] (2\%) the mixed combination G2P [4] / P [6] and G2/ G3P [4] were at 6\%. In 2014, the most predominate genotype was G1P [8] (93\%) followed by G1P [6] (4\%), G2P [4] (2\%) and G3P [8] (1\%) which accounted for very few specimens as illustrated in figure 5 below.

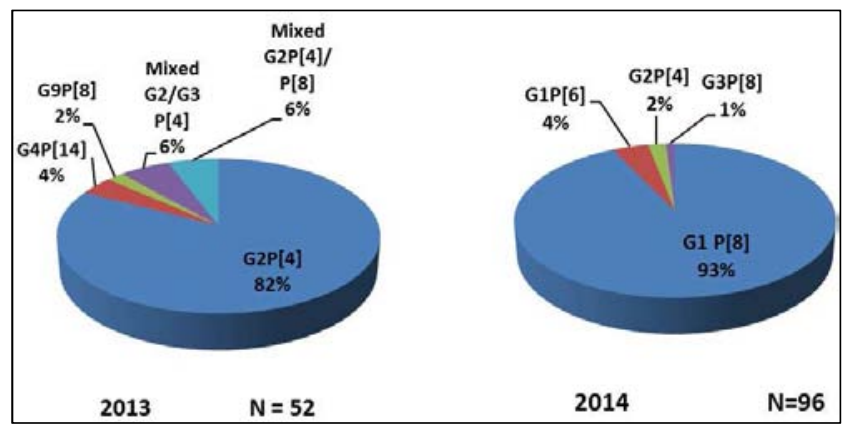

Figure 5. Rotavirus genotypes circulating in the sentinel sites, $2013-2014$, Swaziland.

\section{Discussion}

In Swaziland, Rotavirus infects young children and is a common cause of diarrhoea hospitalization. Our findings have shown that $91 \%$ of infected children are $<2$ years of age with the highest prevalence $(44.7 \%)$ between $6-11$ months old which is comparable with previous studies in Zimbabwe, Uganda and Nigeria [9-11]. This onset of infection correlates very well with the decline of maternally acquired antibodies that disappear around 5 months of age.

Many of the infections were severe with $87.5 \%$ of children experiencing at least some level of dehydration $(53.5 \%$ severe dehydration, $34 \%$ some dehydration and $0.6 \%$ in shock) and $84.3 \%$ received intravenous fluids for rehydration. The reason for managing most of the patients with IV fluids can be due to the high occurrence of vomiting $(86.8 \%)$ among the rotavirus positives in the study population. These children would benefit from rotavirus vaccination program which is a more cost effective intervention.

This study found that all cause acute gastroenteritis and rotavirus diarrhoea were reported mainly in the dry cool seasons of June - August where more than $90 \%$ of the cases occurred. In temperate climates, rotavirus diarrhoea is predominantly a winter disease and few or no cases occur other than at the seasonal peak (Ho eta al 1988, LeBaron et al. 1990, Ryan et al. 1996). Similar findings were reported in Zimbabwe [9] which has a similar temperate climate like Swaziland.

Our study did not observe statistically significant difference between boys and girls for rotavirus gastroenteritis which is similar to Ethiopia and Togo [5, 14]. Some studies have found males to be more affected by rotavirus gastroenteritis $[10,11]$ while other reported females to be more affected [18]

Though high rates of mortality are reported in developing countries, the case fatality among the study population in Swaziland was lower which is similar to the study findings in 
Nigeria [11]. Being a hospital based study that involved only hospitalized children, it is not likely to be representative of the situation in other communities where health facilities and hospitals are not readily accessible and this is one limitation of the study.

Genotype data: Interpretation of findings and implications

In a given year, the incidence of individual genotypes can vary from region to region and multiple G-types and P-types can co-circulate within the same region (Glass, 2006). However, they are unpredictable and it is impossible to predict which genotype will infect children at any specific season per country. In this study the most common genotypes types detected were G1, G2, G3, G4 and G9 associated with $\mathrm{P}$ [4], P [8] and P [6] between year 2013 to 2014 in Swaziland were among the globally detected genotypes combination (Santos and Hoshino, 2005) though a variation per year of the predominating genotypes was observed, G2P [4] at (82\%) in 2013 and G1P [8] at (93\%) in 2014 similar other studies conducted in South Africa (Seheri, et al 2010). This implies the current rotavirus vaccines available globally, when introduced in the country will reduce diarrheal disease caused by rotavirus infection.

\section{Conclusion}

In conclusion, the study showed the high prevalence of severe rotavirus infection in Swaziland among children hospitalized for gastroenteritis. The findings advocate the need for introduction of rotavirus vaccine in to the routine immunization program. Once a rotavirus vaccine is introduced in to the routine immunization program, such surveillance data would allow reliable and rapid assessment of the success or failure of the vaccination program.

\section{Acknowledgements}

The authors would like to thank the Ministry of Health of Swaziland, World health Organization and other partners for material and technical support to the rotavirus sentinel surveillance system which produced these results. The clinical and laboratory staffs of Mbabane Government Referral Hospital, National Reference Laboratory (NRL) and Raleigh Fitkin Memorial hospital are highly appreciated for collecting the surveillance data.

The authors also appreciate the support from MRC Diarrhoeal pathogens research unit, University of Limpopo (MEDUNSA) in genotyping the samples.

\section{Disclaimer}

The findings and conclusions in this report are those of the authors and do not necessarily represent the official position of the World Health Organization (WHO).

\section{Competing Interests}

No competing interest

\section{References}

[1] Luil L, Johnson HL, Cousens S, et al. Global, regional and National causes of child mortality: an updated systematic analysis for 2010 with time trends since 2000. Lancet, 2012; 379: $2151-2161$.

[2] Kotloff KL, Nataro JP, Blackwelder WC, et al. Burden and aetiology of diarrhoeal diseases in infants and young children in developing countries (the Global Enteric Multicenter Study, GEMS): a prospective, case control study, Lancet. 2013; 382: $209-222$.

[3] Tate et al., 2011 Tate SV. Chitambar DSD. (2011). Intragenotypic diversity in the VP4 encoding genes of rotavirus strains circulating in adolescent and adult cases of acute gastroenteritis in Pune, Western India: 1993 to 1996 and 2004 to 2007. Journal Molecular Virology. 3: 53-62.

[4] Tate JE, Burton AH, Boschi -Pinto C, et al; WHO coordinated Global Rotavirus Surveillance Network. 2008 estimate of worldwide rotavirus associated mortality in children younger than 5 years before the introduction of universal rotavirus vaccination programmes, a systematic review and meta analysis. Lancet Infect Dis 2012; 12: 136 - 141.

[5] Abebe A, Teka T, Kassa T, et al. Hospital-based surveillance for rotavirus gastroenteritis in children younger than 5 years of age in Ethiopia 2011. Pediatr Infect Dis J 2014, 33 (Suppl 1): S28-S33.

[6] Enweronu-Laryea CC, Sagoe KWC, Mwenda JM, et al Severe virus gastroenteritis in children less than 5 years in southern Ghana 2011. Pediatr Infect Dis J 2014; 33 (Suppl 1): S9-S13.

[7] Khagayi S, Burton DC, Onkoba R, et al. High burden of rotavirus teritis in young children in rural Western Kenya, 2010-2011. Pediatr Dis J 2014; 33 (Suppl 1) S34-S40.

[8] Kiulia NM, Nyaga MM, Seheri ML, et al. Rotavirus G and P typing in the Eastern region of Kenya; predominance of G9 and emergence of G12 genotypes. Pediatr Infect Dis J. 2014; 33 (Suppl 1); S85-S8.

[9] Mukaratirwa A, Berejena C, Nziramasanga P, et al. Epidemiology genotypic characteristics of rotavirus strains detected in children years of age with gastroenteritis treated at 3 pediatric hospitals in during 2008-2011. Pediatr Infect Dis J 2014; 33 (Suppl 1) S45-S48.

[10] Odiit A, Mulindwa A, Nalum, ansi E, et al. Rotavirus prevalence and genotypes among children younger than 5 years with acute diarrhea at mualgo National Referral Hospital, Kampala, Uganda. Pediatr Infect Dis J. 2014; 33 (Suppl 1): S41 - S44.

[11] Tagbo Bn, Mwenda JM, Armah G, et al. Epidemiology of rotavirus diarrhea among younger than 5 years in Enugu, South East, Nigeria. Pediatr Infect Dis J. 2014; 33 (Suppl 1): s19-S22.

[12] Mustafa A, Makki A, Sidding O, et al. Baseline burden of rotavirus disease in Sudan to monitor the impact of vaccination. Pediatr infect Dis J. 2014; 33 (Suppl 1): S23-S27.

[13] Pursem VN, Peeroo C, Mangar T, et al. Epidemiology of rotavirus diarrhea and diversity of rotavirus strains among children less than 5 years of age with acute gastroenteritis in Mauritius; June 2008 to December 2010. Pediatr Infect Dis J. 2014; 33 (Suppl 1): S49-S53. 
[14] Tsolenynu E, Seheri M, Dagnra A, et al. Surveillance for rotavirus gastroenteritis in children less than 5 years of age in Togo. Pediatr Infect Dis J. 2014; 33 (Suppl 1): S14-S18.

[15] World Health Organization, African rotavirus surveillance network, Manual, Congo Brazzaville, WHO, 2009.

[16] Grimwood K, Lambert SB, Milne RJ. Rotavirus infections and vaccines: burden of illness and potential impact of vaccination. pediatr drugs. 2010; 12: 235-236.

[17] Rotavirus vaccine: an update. Weekly epidemiological record/health section of the secretariat of the League of Nations. Wkly Epidemiological Rec. 2009; 84: 533 - 540.
[18] Rodrigues A, de Varvalho M, Monteiro S etal. Hospital surveillance of rotavirus infection and nosocomial transmission of rotavirus disease among children in GuineaBissau. Paediatrics Infect Dis J. 2007; 26; 233 - 237.

[19] Gouvea V., Glass R. I., Woods P., Taniguchi K., Clark H. F., Forrester B., Fang Z. Y.(1990). Polymerase chain reaction amplification and typing of rotavirus nucleic acid from stool specimens. Journal of Clinical Microbiology 28, 276-282.

[20] Gentsch J. R., Glass R. I., Woods P., Gouvea V., Gorziglia M., Flores J., Das B. K. and Bhan M. K.(1992). Identification of group A rotavirus gene 4 types by polymerase chain reaction. Journal of clinical microbiology 30, 1365-1373. 